\title{
Impact of temporal upscaling and chemical transport model horizontal resolution on reducing ozone exposure misclassification
}

\author{
Yadong Xu ${ }^{a}$, Marc L. Serre a, Jeanette M. Reyes a , William Vizuete ${ }^{\text {a, * }}$ \\ a Department of Environmental Sciences and Engineering, UNC, 135 Dauer Drive, Chapel Hill NC27599-7431, USA
}

\section{H I G H L I G H T S}

- A BME framework that integrates ozone observations and CTM model predictions.

- Compared the impact of multiple temporal upscaling methods.

- Finer CTM horizontal resolution can improve BME estimates.

- BME estimates improved estimation accuracy and spatial variability.

\section{A R T I C L E I N F O}

Article history:

Received 11 April 2017

Received in revised form

13 July 2017

Accepted 17 July 2017

Available online 18 July 2017

Keywords:

Bayesian Maximum Entropy

Ozone

Data fusion

Chemical transport model

\begin{abstract}
A B S T R A C T
We have developed a Bayesian Maximum Entropy (BME) framework that integrates observations from a surface monitoring network and predictions from a Chemical Transport Model (CTM) to create improved exposure estimates that can be resolved into any spatial and temporal resolution. The flexibility of the framework allows for input of data in any choice of time scales and CTM predictions of any spatial resolution with varying associated degrees of estimation error and cost in terms of implementation and computation. This study quantifies the impact on exposure estimation error due to these choices by first comparing estimations errors when BME relied on ozone concentration data either as an hourly average, the daily maximum 8-h average (DM8A), or the daily 24-h average (D24A). Our analysis found that the use of DM8A and D24A data, although less computationally intensive, reduced estimation error more when compared to the use of hourly data. This was primarily due to the poorer CTM model performance in the hourly average predicted ozone. Our second analysis compared spatial variability and estimation errors when BME relied on CTM predictions with a grid cell resolution of $12 \times 12 \mathrm{~km}^{2}$ versus a coarser resolution of $36 \times 36 \mathrm{~km}^{2}$. Our analysis found that integrating the finer grid resolution CTM predictions not only reduced estimation error, but also increased the spatial variability in daily ozone estimates by 5 times. This improvement was due to the improved spatial gradients and model performance found in the finer resolved CTM simulation. The integration of observational and model predictions that is permitted in a BME framework continues to be a powerful approach for improving exposure estimates of ambient air pollution. The results of this analysis demonstrate the importance of also understanding model performance variability and its implications on exposure error.
\end{abstract}

\section{Introduction}

Environmental epidemiologists and exposure scientists require accurate ambient ozone estimates at fine spatial and temporal resolutions to assess human risk to ozone exposure at national or

\footnotetext{
* Corresponding author.

E-mail addresses: yadongx@live.unc.edu (Y. Xu), marc_serre@unc.edu (M.L. Serre), reyesjm@live.unc.edu (J.M. Reyes), vizuete@unc.edu, airquality@unc. edu (W. Vizuete).
}

regional scales. Most air pollution epidemiologic investigations, however, rely on ambient ozone estimates generated solely from air quality monitoring networks (Jerrett et al., 2009; Franklin and Schwartz, 2008; Breton et al., 2012; Pride et al., 2015; Neidell and Kinney, 2010), such as the Air Quality System (AQS) operated by the U.S. Environmental Protection Agency (EPA). The AQS monitoring network has constraints in its geographic and temporal coverage, especially for the ozone-monitoring network. In addition to hourly averages and a sparse national coverage, the AQS network has less than half of its ozone sites operating year round. Only 
during the summer months is the network fully operational producing higher uncertainty for exposure estimates during winter months or for time-weighted averages that include these measurements. Pollutant predictions from a chemical transport model (CTM) are another useful environmental data source to generate exposure estimates. CTM model predictions have the advantage of having continuous spatial coverage, but sometimes are limited in their temporal coverage. Furthermore, there is inherent error and uncertainty in CTM model outputs that can be difficult to quantify. The continued need for ambient air pollution estimates with longer time periods and larger spatial domain has pushed the exposure community to find ways to combine these two data sources in hopes of achieving improved estimation accuracy.

The Bayesian Maximum Entropy (BME) estimation framework has been shown to combine model predictions and observational data to improve exposure estimates (Xu et al., 2016). The flexibility of the framework allows for input of data at any time scale. This choice, however, can have large implications on the cost of the BME implementation and computation time. For example, ozone concentration predicted from the CTM and measured from a surface monitor could be input into BME either as an hourly or daily 24-h average (D24A). If the hourly average is used, then there can be a significant increase in CPU time when compared with using daily values. Using the hourly average, however, retains the detailed diurnal information such as the timing and magnitude of the hourly peak concentrations. This type of information is critical for exposure risk assessment models such as the Air Pollutants Exposure (APEX) Model (EPA, 2014; EPA, 2013). There is a current need for evidence to justify the increased costs associated with choices in time-weighted averages, yet no studies exist investigating how it could affect estimation error. In addition to choices of time averages, the BME framework can accept input from a CTM at any spatial resolution. The available CTM simulations for the continental United States have increased horizontal grid cell resolutions in recent years, requiring greater computational costs, but resulting in more accurate predictions of pollutant concentrations (Appel et al., 2012a; Tessum et al., 2015). The peer-reviewed literature has not explored how much this improvement in CTM model performance can contribute to improving the accuracy of the BME estimation.

The goal of our study is to investigate the extent of the impact that various options in time weighted averaging and CTM horizontal resolution has on the BME exposure estimation error and the capacity of capturing the spatial variability for daily ozone for the year 2005. We will rely on hourly ozone observational data from the AQS database maintained by the U.S. EPA. We will also use continental scale CTM model predictions of hourly ozone concentrations predicted by the Comprehensive Air Quality Model with extensions (CAMx). The CAMx modeling system has a spatial grid cell resolution of $36 \times 36 \mathrm{~km}^{2}$ covering the continental U.S. and a $12 \times 12 \mathrm{~km}^{2}$ grid cell resolution domain covering the Eastern U.S. We will determine the changes in estimation error on daily ozone and determine whether it is worth the cost in using the hourly ozone data as opposed to the DM8A, or D24A daily data. Also, this is the first work to quantify the improvement of using finer scale CTM predictions in our BME framework.

\section{Data sources}

\subsection{Observational data}

The ozone observational data are observed hourly ozone monitoring data sampled from 1179 sites in the continental U.S. for the year 2005 downloaded from the AQS database maintained by the U.S. EPA. The majority of these AQS sites are located in the populated area of the eastern and western U.S. while others are located in very sparse areas in the central U.S. such as the states of Colorado, Kansas, Nebraska, New Mexico, North Dakota and South Dakota (SI Fig. 1s). The highest hourly ozone reported in year 2005 was $182 \mathrm{ppb}$. We see large national-scale spatial variability in hourly ozone concentrations, with annual mean on each site range from 9.6 ppb to $58.4 \mathrm{ppb}$ (SI Fig. 1s and 2s). In 2005, only 625 AQS sites operated year-round, with ozone hourly monitoring data available for both the ozone season (May-September) and other months of the year while the other 554 AQS sites only had monitoring data available for the ozone season. The hourly ozone concentrations during summer months (May, June, July and August) are substantially higher (a median value at $33.0 \mathrm{ppb}$ ) than the ones, during the winter months (January, February, November and December) (a median value at $20.0 \mathrm{ppb}$ ) (Fig. $3 \mathrm{~s}$ and Table 1s). Details on the processing and quality assurance of this data can be found in Xu et al. (2016).

\subsection{Chemical transport model}

The CTM model predictions consist of hourly ozone concentrations for the entire year of 2005 predicted by the CAMx modeling system. Model prediction have a spatial grid cell resolution of $36 \times 36 \mathrm{~km}^{2}$ covering the continental U.S. and a $12 \times 12 \mathrm{~km}^{2}$ grid cell resolution domain covering the Eastern U.S as shown in Fig. $4 \mathrm{~s}$. These model simulations were conducted as part of the air quality modeling performed to support the Final Transport Rule by the U.S. EPA. The simulations for the year 2005 were used as a base year for several other emissions sensitivity scenarios. Detailed model configurations and evaluation are documented in the Air Quality Modeling Final Rule Technical Support Document by the U.S. EPA (SI Appendix 1). According to the model performance evaluation reported by the U.S. EPA (Appendix A of the document titled "Air Quality Modeling Final Rule Technical Support Document"), overall, the model performance results for the 8-h daily maximum ozone concentrations from the 2005 CAMx model simulations are within the range, or close to those found in recent regional ozone model applications (Appel et al., 2007; Tesche et al., 2006; Appel et al., 2009; Foley et al., 2009).

Before using these CTM predictions, we quantified the spatial and temporal variability in model performance. For this evaluation each observed hourly concentration $z_{j}$ (the subscript $j$ refers to space/time location of observed data) is paired with its corresponding CTM prediction value $\tilde{z}_{j}$, and the error for the observation-prediction pair is defined as $e_{j}=\tilde{z}_{j}-z_{j}$. To evaluate the CTM model performance over a given space time region $R$ of interest, we calculate commonly used error statistics such as the Mean Prediction Error (ME), the Standard deviation of the prediction error (SE), the mean normalized bias (MNB), the mean normalized gross error (MNGE), the normalized mean bias factor (NMBF) and the normalized mean absolute error factor (NMAEF) (Yu et al., ) as defined in SI equations (7s)-(12s).

Detailed results of the nationwide model performance analysis of this CTM simulation are provided in SI section 1. In brief, we find that overall the CAMx predictions using the $12 \times 12 \mathrm{~km}$ grid cell resolution domain has a substantially lower over-prediction (median $\mathrm{ME}=+3.4 \mathrm{ppb}$ ) than predictions relying on a $36 \times 36 \mathrm{~km}$ grid cell resolution (median $\mathrm{ME}=+6.1 \mathrm{ppb}$ ) as shown in Table $2 \mathrm{~s}$. There are clear geographical trends in the variability of these error statistics: urban cities located in the east and west coast tend to have higher over-prediction errors (i.e. higher ME) and higher imprecision (i.e. higher SE) than sites located in the central United States as shown in Figs. 7s-10s. The seasonal difference in model performance was also analyzed by recalculating the error statistics at each site separately for the summer (May, June, July and August) and 
winter (November, December, January and February). The overprediction bias is noticeably higher in the summer, with the median ME for the summer equal to $4.1 \mathrm{ppb}$ compared to $2.3 \mathrm{ppb}$ for the winter. The median MNB (237\%) and MNGE (267\%) values for the winter are much higher than for the summer with the median MNB at $129 \%$ and the median MNGE at $143 \%$. These results indicate that the CTM model's difficulty at predicting lower ozone concentrations observed in the winter.

Generally, the CTM reproduces hourly ozone concentrations with much higher prediction errors than the daily DM8A and D24A ozone values. For example, in the CAMx simulation with $12 \times 12 \mathrm{~km}^{2}$ grid cell resolution, the median SE for hourly ozone is $13.2 \mathrm{ppb}$, which is much higher than the ones for DM8A at $9.4 \mathrm{ppb}$ and for D24A at $7.9 \mathrm{ppb}$ as shown in Table 2s. The differences in MNB and MNGE are even greater between the hourly and daily metrics, with the median MNB at $145 \%$ for hourly ozone compared to for DM8A at $9.3 \%$ and for D24A at $19.8 \%$. This can be explained by the fact that CTM models tend to severely over-predict nighttime hourly ozone concentrations at very low values and under-predict peak hourly ozone concentrations (Appel et al., 2007; Tesche et al., 2006; Appel et al., 2012b). According to the results from the more statistically robust measures of NMBF and NMAEF, the CAMx simulations still performed better for the daily DM8A, with a median NMAEF at $17 \%$, and D24A, with a median NMAEF at $25 \%$, than for the hourly concentrations with a median NMAEF at $37 \%$.

\section{Methods}

\subsection{BME estimation methodology}

A brief review of the BME framework used in our previous work to generate daily ozone estimates can be found in SI section 1 and more detailed descriptions in Xu et al. (2016). In short, the AQS observed data are first paired with CTM predicted ozone concentrations in space/time. Then, a localized bias-corrected CTM data are constructed as the soft data (i.e. secondary information with uncertainties) for the BME estimation. The soft data are produced via the Regionalized Air Quality Model Performance (RAMP) method where CTM predictions are weighted according to model performance that varies across space and time. Next, a transformation of the data was completed by removing an offset defined as an exponential kernel smoothing of the data. The exponential kernel smoothing was set so that the offset captured the spatial variability of the data over intermediate spatial distances and intermediate time scales. Finally, a space-time covariance model is built based on a 3-term exponential/exponential/cosine model that was used to characterize the space/time autocorrelation in the offset removed ozone data.

To conduct the final BME interpolation we: 1) use the Maximum Entropy principle of information theory to process the general knowledge base $(\mathrm{G}-\mathrm{KB})$ consisting of the mean function and the covariance function of ozone 2) integrates the site specific knowledge base (S-KB) using an epistemic Bayesian conditionalization rule to create a BME posterior probability density function (PDF) $f_{K}$ characterizing the value $x_{k}$ taken by the space/time random field $X(\boldsymbol{p})$ at any estimation point $\boldsymbol{p}_{k}=\left(\boldsymbol{s}_{k}, t_{k}\right)$ of interest, and 3) computes space/time estimates based on the BME posterior PDF. The SKB consisted of observations treated as hard data (an error-free proxy) and localized bias-corrected CTM data treated as soft data.

\subsubsection{Soft data construction}

The RAMP method was used to produce the BME soft data. The RAMP evaluation analysis is conducted in two stages. In the first stage we analyze CTM performance at specific monitoring stations, and in the second stage we perform an inverse weighted distance interpolation to assess CTM performance away from monitoring stations. In Xu et al. (2016), we described the details of generating the daily soft data through the RAMP method. Here we focus on describing how to produce the hourly soft data from the CTM model predictions.

In the first stage, we focus specifically on each monitoring site $s_{n}$, and for each monitoring site we pool the hourly observationprediction pairs $\left(z_{j}, \tilde{z}_{j}\right)$ that are within a time tolerance $\Delta T$ of a particular time of interest $t$. These pairs are highly relevant to the location $\boldsymbol{s}_{n}$ where the monitoring station is located. To be consistent with our previous study, we keep the same $\Delta T=120$ days for the hourly ozone. Examples of two selected sites are shown in Fig. 1. We stratify the pairs in 10 percentile bins of increasing predicted values $\tilde{z}_{j}$, and for each bin $\tilde{z}_{b}$ we calculate the mean $\widehat{\lambda}_{1}\left(\tilde{z}_{b}, \boldsymbol{s}_{n}, t\right)$ and variance $\widehat{\lambda}_{2}\left(\tilde{z}_{b}, \boldsymbol{s}_{n}, t\right)$ of observed values (see detailed equations in Xu et al. (2016)).

In the second stage we obtain the mean $\lambda_{1}\left(\tilde{z}_{i}, \boldsymbol{p}_{i}\right)$ and variance $\lambda_{2}\left(\tilde{z}_{i}, \boldsymbol{p}_{i}\right)$ for actual predicted hourly values $\tilde{z}_{i}$ at space/time grid point $\boldsymbol{p}_{i}=\left(\boldsymbol{s}_{i}, t_{i}\right)$. We first perform a linear interpolation/extrapolation of the $\widehat{\lambda}_{1}\left(\tilde{z}_{b}, \boldsymbol{s}_{n}, t_{i}\right)$ and $\widehat{\lambda}_{2}\left(\tilde{z}_{b}, \boldsymbol{s}_{n}, t_{i}\right)$ values at each monitoring site to obtain $\hat{\lambda}_{1}\left(\tilde{z}_{i}, \boldsymbol{s}_{n}, t_{i}\right)$ and $\hat{\lambda}_{2}\left(\tilde{z}_{i}, \boldsymbol{s}_{n}, t_{i}\right)$ at the predicted values. Then we use inverse weighted distance to do a spatial interpolation of these values from the neighboring monitoring stations to obtain $\widehat{\lambda}_{1}\left(\tilde{z}_{i}, \boldsymbol{p}_{i}\right)$ and $\hat{\lambda}_{2}\left(\tilde{z}_{i}, \boldsymbol{p}_{i}\right)$ at $\boldsymbol{p}_{i}$.

\subsubsection{Offset analysis}

The offset is used to transform the hourly ozone data into residual offset-removed data. The ozone offset $o_{Z}\left(\boldsymbol{p}_{i}\right)$ at an arbitrary location $\boldsymbol{p}_{i}$ is obtained using an exponential kernel smoothing (Lee et al., 2012) of the surrounding observed ozone data. Several sets of kernel smoothing ranges, including the spatial range $a_{r}$ and the temporal range $a_{t}$, are investigated. An optimal set of kernel smoothing ranges $\left(a_{r}=50 \mathrm{~km}\right.$ and $a_{t}=10$ day $)$ were chosen to ensure the transformed data has a low variance so that the geostatistical estimation error variance is minimized, while retaining high autocorrelation to ensure that neighboring data locations are informative at the estimation location. These offset ranges are also consistent with what we used for the daily metrics. (See SI section 4 for details).

\subsubsection{Space-time covariance model}

The covariance model for the homogeneous/stationary space/ time random field S/TRF $X(\boldsymbol{p})$ is developed from the experimental covariance of the transformed observational data $\boldsymbol{x}_{j}=\boldsymbol{z}_{j}-o_{Z}\left(\boldsymbol{p}_{j}\right)$. The experimental covariance value for a spatial lag $r$ and a temporal lag $\tau$ is calculated and plotted separately to facilitate the visualization of the space/time covariance models. Several covariance models were attempted and evaluated. A 3-structured exponential/ exponential/cosine covariance model was chosen for the subsequent BME analysis (see SI section 4 for details).

The formula of the 3-structured exponential/cosine covariance model is given by:

$$
\begin{aligned}
c_{X}(r, \tau)= & c_{0}\left[\alpha \exp \left(\frac{-3 r}{a_{r 1}}\right) \exp \left(\frac{-3 \tau}{a_{t 1}}\right)+\beta \exp \left(\frac{-3 r}{a_{r 2}}\right) \exp \left(\frac{-3 \tau}{a_{t 1}}\right)\right. \\
& \left.+(1-\alpha-\beta) \exp \left(\frac{-3 r}{a_{r 2}}\right) \cos \left(\pi \frac{\tau}{a_{t 2}}\right)\right]
\end{aligned}
$$

Where $C_{0}$ is the sill (variance), $a_{r 1}$ and $a_{r 2}$ are the spatial ranges and $a_{t 1}$ and $a_{t 2}$ are the temporal ranges, and $\alpha, \beta$ and $(1-\alpha-\beta)$ are the proportions of variability contributed by the first, second and third 

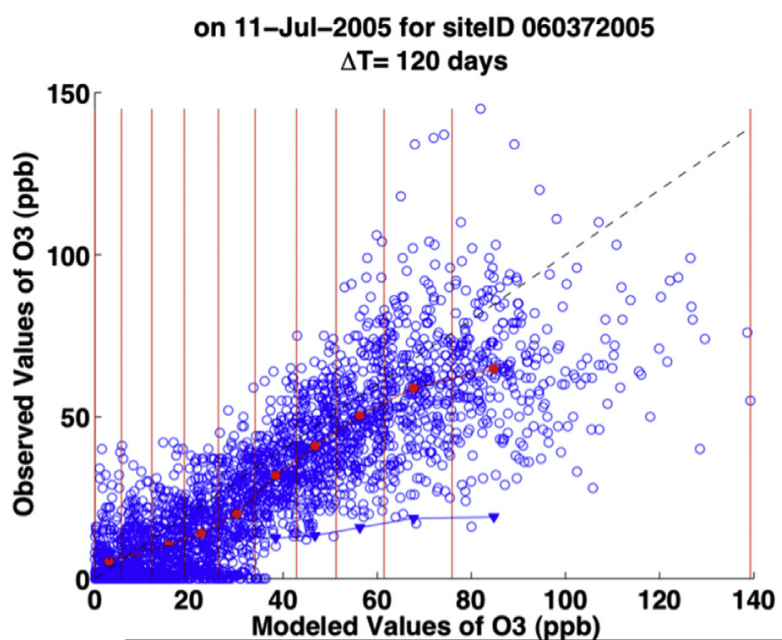

on 11-Jul-2005 for sitelD 120713002 $\Delta \mathrm{T}=120$ days

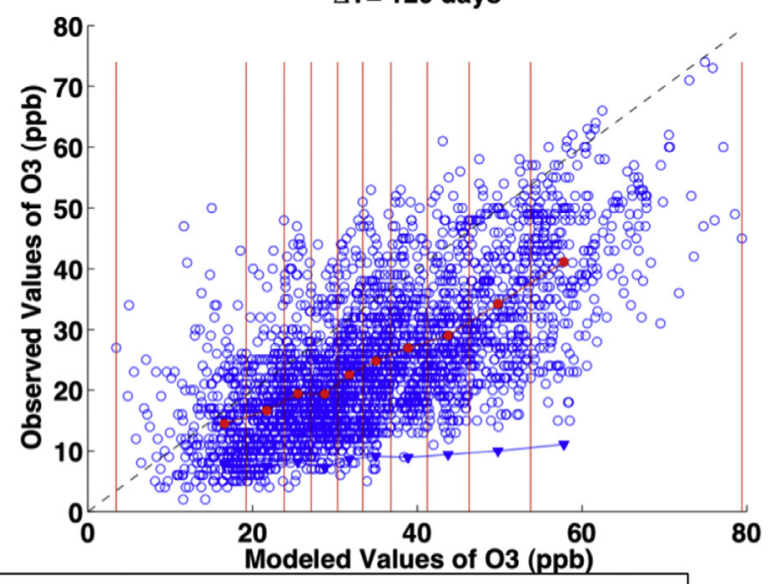

$\circ \quad$ paired hourly $03-$ the bins $\longrightarrow$ the mean in each bin $\longrightarrow-$ SD of the Obs in each bin

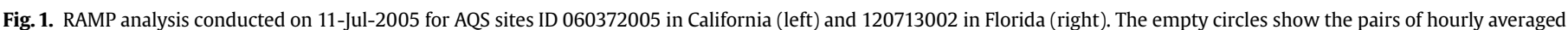

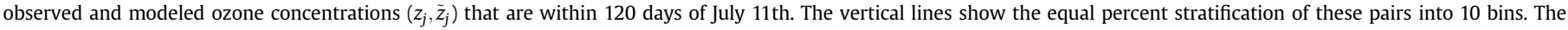

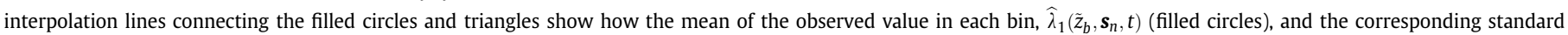
deviation, $\sqrt{\widehat{\lambda}_{2}\left(\tilde{z}_{b}, \boldsymbol{s}_{n}, t\right)}$ (filled triangles) change as a function of the average modeled value $\tilde{z}_{b}$ in that bin.

covariance structure, respectively. The parameters $\alpha, \beta, a_{r 1}, a_{r 2}, a_{t 1}$, and $a_{t 2}$ obtained by least square fitting for each offset are shown in SI Table 3s.

Compared with the covariance models for the daily metrics $\mathrm{Xu}$ et al. (2016), the major difference of the covariance model for the hourly ozone is the cosine function in the third structure of equation (1). This function represents the diurnal pattern occurring in the observational ozone data, which was altered by the aggregation process for the daily metrics.

\subsection{Temporal upscaling and soft data scenarios}

The BME methodology can input averages at any time scale from the CTM predictions and observational data for its estimation. In our analysis the finest resolved temporal average available is hourly that could be temporally upscaled to make daily average BME estimates. For this analysis we created three sets of input files for our BME framework with different temporal averages: hourly, DM8A and D24A. For each of these we have the choice of using soft data with observations or not. When CTM predictions were used for the soft data construction it was chosen from the simulation with the finest grid cell resolution. The combination of input temporal averages and choice of soft data produced the 8 different BME scenarios listed in Table 1. For example, in estimation scenario DM8A-3 we averaged the hourly data from the observations and from CTM predictions into a DM8A temporal average and then used it as input in our BME framework. We then used BME to produce DM8A estimations of ozone. These estimates were then compared with estimates of DM8A made by BME when using hourly data as an input
(DM8A-4). The computational cost of generating hourly estimates directly from BME is substantially higher. For example, to map the continental US at $36 \times 36 \mathrm{~km}^{2}$ resolution with DM8A estimates, the average CPU time for scenario DM8A-4 required $3403 \mathrm{~h}$ compared to only $13.4 \mathrm{~h}$ for scenario DM8A-3 as shown in Tables $7 \mathrm{~s}-1$

\subsection{Validation analysis method}

A validation analysis is used to assess the accuracy of BME estimations due to changes in input data at different time scales or CTM model resolution. In a validation analysis each observed value $z_{j}$ at space/time point $\boldsymbol{p}_{j}=\left(\boldsymbol{s}_{j}, t_{j}\right)$ is compared with the corresponding ozone concentration $z_{j}^{*}$ re-estimated using only noncollocated data outside of a radius $r_{v}$ of $\boldsymbol{s}_{j}$.

The validation error, which is the difference between each reestimated value $z_{j}^{*}\left(r_{v}\right)$ and observed value $z_{j}$ is defined as $e_{j}^{*}\left(r_{v}\right)=z_{j}^{*}\left(r_{v}\right)-z_{j}$. The estimation accuracy is quantified based on statistics of these estimation errors, which is a function of the validation radius $r_{v}$. They consist of the Root Mean Square Error RMSE (ppb), the $R^{2}$ (unitless), the Mean Normalized Bias MNB (\%) and the Mean Normalized Gross Error MNGE (\%) between observations and re-estimated values, calculated as a function of $r_{v}$ shown as below.

$$
\operatorname{RMSE}\left(r_{v}\right)=\sqrt{\frac{1}{N_{o}} \sum_{j=1}^{N_{o}}\left(z_{j}^{*}\left(r_{v}\right)-z_{j}\right)^{2}}
$$

Table 1

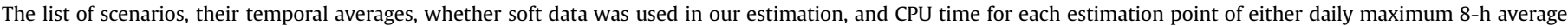
(DM8A) or daily 24-h average (D24A) ozone concentrations.

\begin{tabular}{|c|c|c|c|c|c|c|c|}
\hline \multicolumn{4}{|c|}{ To estimate DM8A } & \multicolumn{4}{|c|}{ To estimate D24A } \\
\hline Scenario name & Input temporal average & Soft data & CPU run time (Seconds) & Scenario name & Input temporal average & Soft data & CPU run time (Seconds) \\
\hline DM8A-1 & DM8A & NO & 0.1 & D24A-1 & D24A & NO & 0.1 \\
\hline DM8A-2 & hourly & NO & 2.1 & $\mathrm{D} 24 \mathrm{~A}-2$ & hourly & NO & 2.1 \\
\hline DM8A-3 & DM8A & YES & 2.9 & D24A-3 & $\mathrm{D} 24 \mathrm{~A}$ & YES & 2.9 \\
\hline DM8A-4 & hourly & YES & 23.1 & D24A-4 & hourly & YES & 23.1 \\
\hline
\end{tabular}


$R^{2}\left(r_{v}\right)=\left(\frac{\sum_{j=1}^{N_{o}}\left(z_{j}^{*}\left(r_{v}\right)-\overline{z^{*}\left(r_{v}\right)}\right)\left(z_{j}-\bar{z}\right)}{\sqrt{\sum_{j=1}^{N_{o}}\left(z_{j}^{*}\left(r_{v}\right)-\overline{z^{*}\left(r_{v}\right)}\right)^{2}} * \sqrt{\sum_{j=1}^{N_{o}}\left(z_{j}-\bar{z}\right)^{2}}}\right)^{2}$

$\operatorname{MNB}\left(r_{v}\right)=\frac{1}{N} \sum_{j=1}^{N_{0}} 100 \% *\left(z_{j}^{*}\left(r_{v}\right)-z_{j}\right) / z_{j}$

$\operatorname{MNGE}\left(r_{v}\right)=\frac{1}{N} \sum_{j=1}^{N_{0}} 100 \% *\left(\left|z_{j}^{*}\left(r_{v}\right)-z_{j}\right| / z_{j}\right)$

\section{Results}

\subsection{Temporal upscaling and estimation accuracy}

\subsubsection{Spatial estimates of ozone}

To visualize differences due to temporal upscaling, the BME framework was used to generate ozone estimates for Jul-11-2005. This day was chosen because it has the highest standard deviation (33.1 ppb) for CTM prediction errors when compared against DM8A from surface monitors. Thus, this day represents an ideal test case given the high heterogeneity of CTM model performance. Fig. 2 shows the BME estimates of daily ozone concentrations for Jul-112005 using either a running 8-h average (DM8A) or a daily average (D24A) as an input (scenarios DM8A-3 and D24A-3). Also shown in the figure are the BME estimates that relied on hourly averages as an input (scenarios DM8A-4 and D24A-4). In all these scenarios the BME framework is using soft data. Regardless of the input temporal average, the final estimates were quite similar and captured the locations of high (above $70 \mathrm{ppb}$ ) and low (below $40 \mathrm{ppb}$ ) ozone. For example, DM8A-3 and DM8A-4 both estimated $70 \mathrm{ppb}$ in Wisconsin and Michigan and values of less than $30 \mathrm{ppb}$ in Georgia and Florida. For the DM8A, the averaged difference (between scenarios DM8A-3 and DM8A-4) across the continental U.S is $4.8 \mathrm{ppb}$ with the largest difference of $32.5 \mathrm{ppb}$ occurring in Kentucky. For the D24A, the averaged difference (between scenarios D24A-3 and D24A-4) is $3.3 \mathrm{ppb}$ with the largest difference of $29.6 \mathrm{ppb}$.

To quantify the influence that the CTM predictions via the soft data had on estimates we generated scenarios DM8A-1, DM8A-2, D24A-1 and D24A-2. In these scenarios BME made ozone estimates without the use of soft data. Our results show almost no impact on estimates of ozone when using either an hourly or longer temporal average (SI section 4.1).

\subsubsection{Validation results for daily ozone estimates}

To compare the predictive capacity between these two temporal upscaling methods we calculated estimation error using our validation analysis method described in section 3.3. The validation analysis was conducted at all 1179 ozone monitoring sites located in the continental US. Table 2 shows validation statistics for the eight scenarios defined in Table 1 . We found that when we first aggregate the hourly data into a daily value (DM8A-3), the estimation accuracy is improved with lower RMSE and a higher $\mathrm{R}^{2}$, when compared to estimates that used hourly averages (DM8A-4). This can be explained by the poorer model performance when using hourly averaged CTM predictions. The uncertainty associated with the hourly ozone model performance is over $40 \%$ higher, in terms of the median SE, compared with DM8A (SI Table 2s). This higher uncertainty in hourly model predictions was transferred into less informative soft data and thus had little impact on the final BME estimates. Further evidence of this is seen when the soft data was not integrated in the BME framework, in scenarios DM8A-1 and DM8A-2, the estimation accuracy were similar with DM8A-1
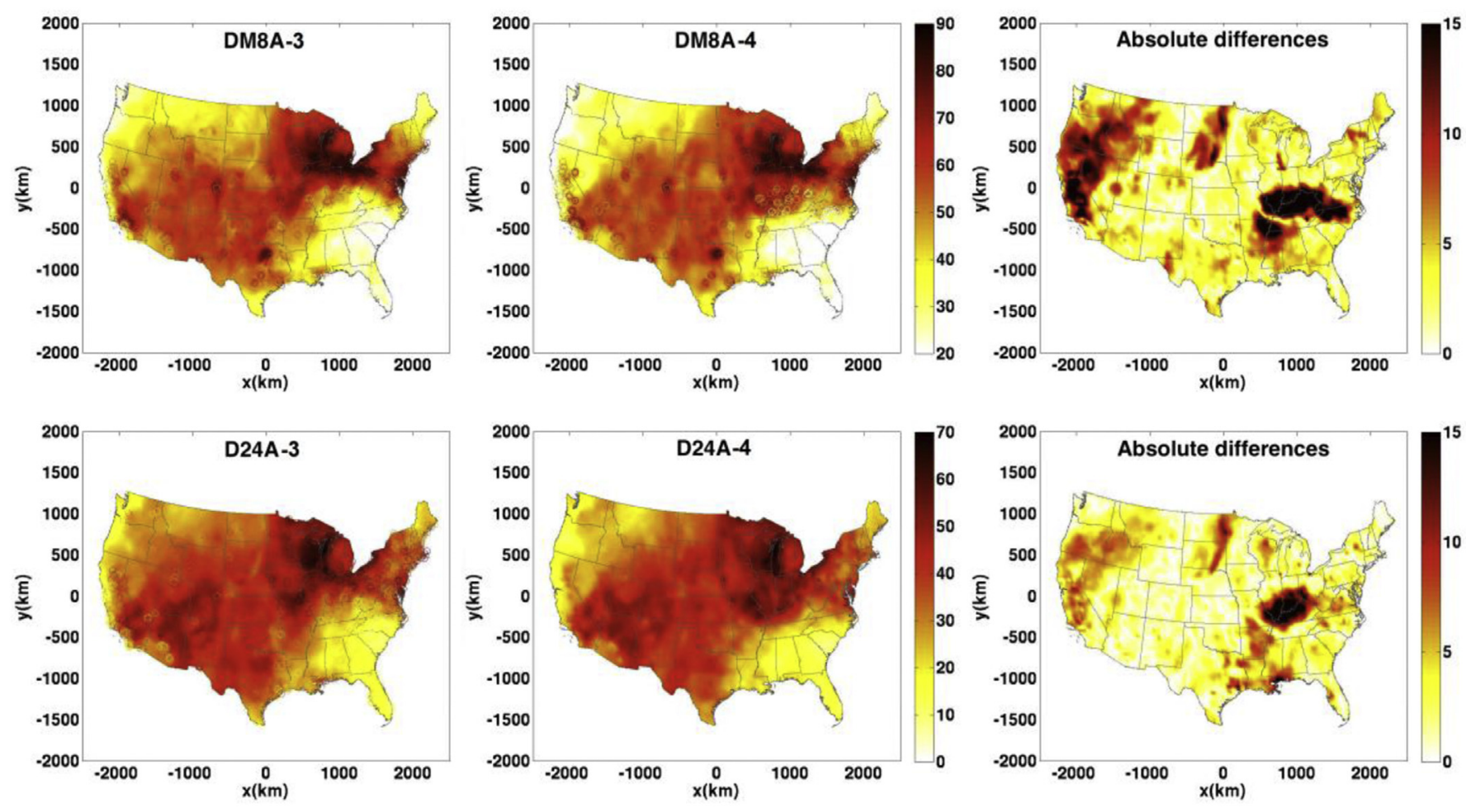

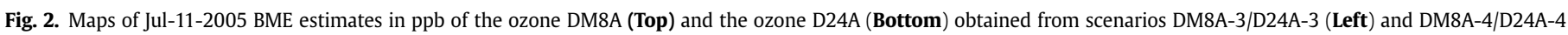
(Middle). Also shown are the absolute differences between DM8A-3 and DM8A-4 (TOP Right), and also between D24A-3 and D24A-4 (Bottom Right). 
Table 2

Validation statistics for daily maximum 8-h (DM8A) and 24-h daily ozone (D24A) for the eight scenarios defined in Table 1

\begin{tabular}{|c|c|c|c|c|c|c|}
\hline \multirow[t]{2}{*}{ Output Time Average } & \multirow[t]{2}{*}{ Input Time Average } & \multirow[t]{2}{*}{ Error statistics } & \multicolumn{4}{|c|}{$r_{v}(\mathbf{k m})^{\mathrm{a}}$} \\
\hline & & & 0 & 36 & 72 & 108 \\
\hline \multirow[t]{9}{*}{ DM8A } & DM8A & $R_{M S E_{D M 8 A-1}}(p p b)^{\mathrm{b}}$ & 5.536 & 6.309 & 6.799 & 7.041 \\
\hline & hourly & $R M S E_{D M 8 A-2}(p p b)^{\mathrm{b}}$ & 5.683 & 6.566 & 7.455 & 7.909 \\
\hline & DM8A & RMSE $_{D M 8 A-3}(p p b)^{\mathrm{b}}$ & 5.445 & 6.109 & 6.531 & 6.732 \\
\hline & hourly & $\operatorname{RMSE}_{D M 8 A-4}(p p b)^{\mathrm{b}}$ & 6.206 & 7.420 & 8.127 & 8.416 \\
\hline & DM8A & $R_{D M 8 A-1}^{2}\left(\right.$ unitless) ${ }^{\mathrm{C}}$ & 0.886 & 0.853 & 0.829 & 0.817 \\
\hline & hourly & $R_{D M 8 A-2}^{2}$ (unitless) ${ }^{\mathrm{C}}$ & 0.884 & 0.845 & 0.803 & 0.781 \\
\hline & DM8A & $R_{D M 8 A-3}^{2}(\text { unitless) })^{\mathrm{C}}$ & 0.893 & 0.866 & 0.849 & 0.841 \\
\hline & hourly & $R_{D M 8 A-4}^{2}\left(\right.$ unitless) ${ }^{\mathrm{C}}$ & 0.882 & 0.846 & 0.816 & 0.805 \\
\hline & $\mathrm{N} / \mathrm{A}$ & $\mathrm{PCR}^{2}{ }_{\text {DM8A-1 }} \rightarrow$ DM8A-3 $(\%)^{\mathrm{d}}$ & 0.726 & 1.602 & 2.407 & 2.936 \\
\hline \multirow[t]{9}{*}{ D24A } & $\mathrm{D} 24 \mathrm{~A}$ & $R{ } E_{D 24 A-1}(p p b)^{\mathrm{b}}$ & 5.705 & 6.178 & 6.303 & 6.422 \\
\hline & hourly & $\operatorname{RMSE}_{D 24 A-2}(p p b)^{\mathrm{b}}$ & 5.722 & 6.170 & 6.292 & 6.455 \\
\hline & $\mathrm{D} 24 \mathrm{~A}$ & $\operatorname{RMSE}_{D 24 A-3}(p p b)^{\mathrm{b}}$ & 5.487 & 5.835 & 5.917 & 6.004 \\
\hline & hourly & $R M S E_{D 24 A-4}(p p b)^{\mathrm{b}}$ & 5.486 & 5.819 & 5.997 & 6.100 \\
\hline & $\mathrm{D} 24 \mathrm{~A}$ & $R_{D 24 A-1}^{2}\left(\right.$ unitless) ${ }^{C}$ & 0.792 & 0.757 & 0.747 & 0.738 \\
\hline & hourly & $R_{D 24 A-2}^{2}$ (unitless) ${ }^{c}$ & 0.792 & 0.759 & 0.749 & 0.738 \\
\hline & $\mathrm{D} 24 \mathrm{~A}$ & $R_{D 24 A-3}^{2}$ (unitless) ${ }^{c}$ & 0.813 & 0.789 & 0.786 & 0.781 \\
\hline & hourly & $R_{D 24 A-4}^{2}\left(\right.$ unitless) ${ }^{\mathrm{C}}$ & 0.809 & 0.789 & 0.777 & 0.770 \\
\hline & $\mathrm{N} / \mathrm{A}$ & $\mathrm{PCR}^{2}{ }_{\mathrm{D} 24 \mathrm{~A}-1 \rightarrow \mathrm{D} 24 \mathrm{~A}-3}(\%)^{\mathrm{d}}$ & 2.642 & 4.338 & 5.190 & 5.898 \\
\hline
\end{tabular}

having a slightly lower RMSE and higher $\mathrm{R}^{2}$. This indicates that using DM8A directly for BME input can achieve higher estimation accuracy when compared with using hourly data.

In the case of D24A, when soft data are used, the estimation accuracy for D24A-3 is generally better than the one for D24A-4 with slightly higher $\mathrm{R}^{2}\left(0.813\right.$ vs 0.809 when $r_{v}$ at 0$)$ (Table 2). Similar to the DM8A scenarios, when not using soft data, the values of RMSE and $\mathrm{R}^{2}$ for D24A-1 and D24A-2 are very similar, with less than $0.3 \%$ of the absolute differences. This confirms again that using daily values directly as BME input is a better option because it improves estimation accuracy and also substantially reduces the computational cost.

The $P C R^{2}$ is the percent change in $R^{2}$ when either using soft data or not between DM8A-1 and DM8A-3. As shown in Table 1 the PCR is consistently positive. This indicates that incorporating CTM predictions can improve the estimation accuracy compared with using the observational data alone. As expected, the percent change in $R^{2}$ increases as the validation radius $r_{v}$ increases (from 0 to $108 \mathrm{~km}$ ), indicating that the soft data from CTM can improve the estimation accuracy even more at the locations far away from any monitoring sites.

\subsection{CTM model grid cell resolution and estimation accuracy}

\subsubsection{Spatial estimates of ozone}

Based on the results shown in section 4.1, we demonstrated the importance of incorporating soft data in the BME analysis and also concluded that using daily averages provides better predictive accuracy and requires less computational resources. Thus, in the second part of our analysis we created four more estimation scenarios to investigate the influence of the CTM horizontal grid cell resolution on our soft data construction and estimation accuracy as shown in Table 3 . We focused solely on a smaller domain where the CTM $36 \times 36 \mathrm{~km}$ and $12 \times 12 \mathrm{~km}$ domains overlapped as shown in the green box in Fig. 1s. In these estimation scenarios the soft data is constructed either with predictions from CTM using the $36 \times 36 \mathrm{~km}$ grid cell resolution domain (scenarios DM8A-5 and D24A-5) or the $12 \times 12 \mathrm{~km}$ grid cell resolution domain (scenarios DM8A-6 and D24A-6).

For this analysis we wanted to target a day that had the largest differences in local spatial variability between the CTM predictions based on the two grid cell resolutions. To capture this spatial variability over a given region $R$ of interest, we define the normalized variance shown in Equation (6). This value is the variance among the ozone concentrations at the grid cells within a region normalized by the squared mean for the ozone concentrations at these grid cells, as defined in the equation as below

$\operatorname{NVAR}(R)=\frac{\sum_{j=1}^{N(R)}\left(\tilde{z}_{j}-\tilde{z}_{\text {average }}(R)\right)^{2}}{N(R)-1} / \tilde{z}_{\text {average }}(R)^{2}$

Where $\tilde{z}_{j}$ is the hourly or daily concentration, $\tilde{z}_{\text {average }}(R)$ is the mean of all the hourly or daily values for the grid cells across region $R$. $N(R)$ is the number of grid cells in region $R$.

Using equation (6) we focused on the area covering the states of Iowa, Illinois, Indiana and Missouri shown in the top panel of Fig. 3. We calculated NVAR for the CTM predictions in box A and box B, each with a size of $144 \times 144 \mathrm{~km}^{2}$. The ratio of NVAR between the $36 \times 36 \mathrm{~km}^{2}$ and the $12 \times 12 \mathrm{~km}^{2}$ CTM predictions was 5.6 for box A and 5.0 for box B. These ratios mean that in these regions the spatial variability captured by the $12 \times 12 \mathrm{~km}^{2}$ CTM predictions is more than 5 times greater than that captured by the $36 \times 36 \mathrm{~km}^{2}$ CTM predictions.

We then calculated the ratio of the normalized variances for boxes $\mathrm{A}$ and $\mathrm{B}$, but for the resulting BME mean estimates shown in bottom panel of Fig. 3. For this calculation we also used an estimation radius $\left(r_{h}\right)$ around each estimation point. This radius means that all observational data within that range is excluded in the BME integration. The normalized variance as a function of estimation radius is shown in Table 4 as well as the ratio between scenario DM8A-5 and DM8A-6. All the ratios listed in the table are greater than 1 , which means that the spatial variability captured by the BME mean estimates obtained using the finer resolution $\left(12 \times 12 \mathrm{~km}^{2}\right)$ CTM predictions was consistently greater than the spatial variability captured by the BME estimates obtained from the coarser $\left(36 \times 36 \mathrm{~km}^{2}\right)$ CTM predictions. We also can see that the ratio of the normalized variances increases from 4.48 to 5.36 in box A (from 1.55 to 3.92 in box B) as the excluding radius expands. This indicates that the influence of the finer grid cell resolution on the capability of BME capturing the spatial variability is greater for those regions where the observational data from the ozone 
Table 3

The list of scenarios and what soft data was used in our estimation of either daily maximum 8-h average (DM8A) or daily 24-h average (D24A) ozone concentrations.

\begin{tabular}{|c|c|c|c|c|c|}
\hline \multicolumn{3}{|c|}{ To estimate DM8A } & \multicolumn{3}{|c|}{ To estimate D24A } \\
\hline Scenario name & Input temporal average & Soft data & Scenario name & Input temporal average & Soft data \\
\hline DM8A-5 & DM8A & CTM_36 km & D24A-5 & D24A & CTM_36 km \\
\hline DM8A-6 & DM8A & CTM_12 km & D24A-6 & D24A & CTM_12 km \\
\hline
\end{tabular}

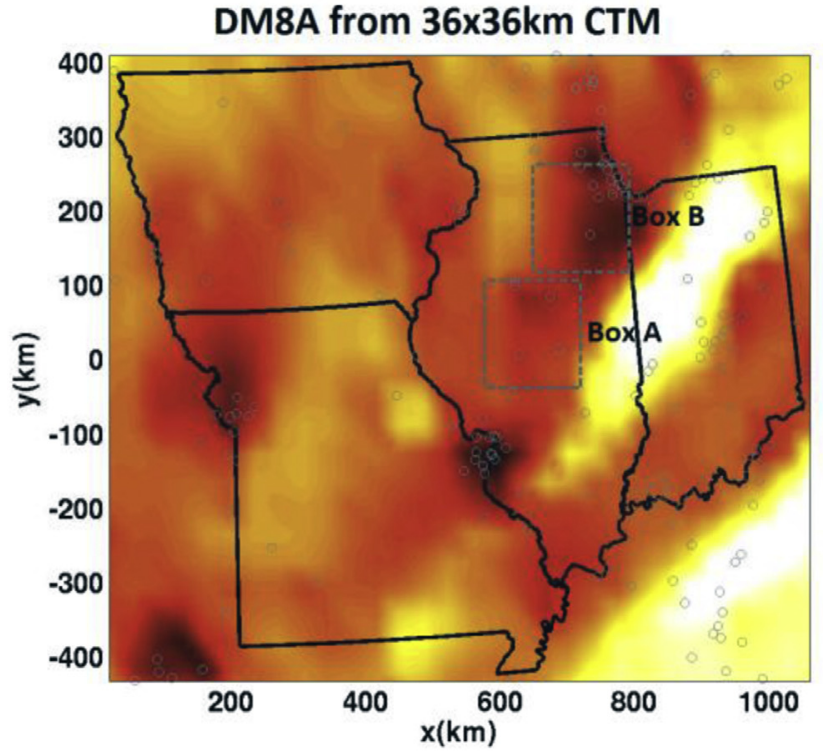

DM8A BME mean using 36x36km soft data

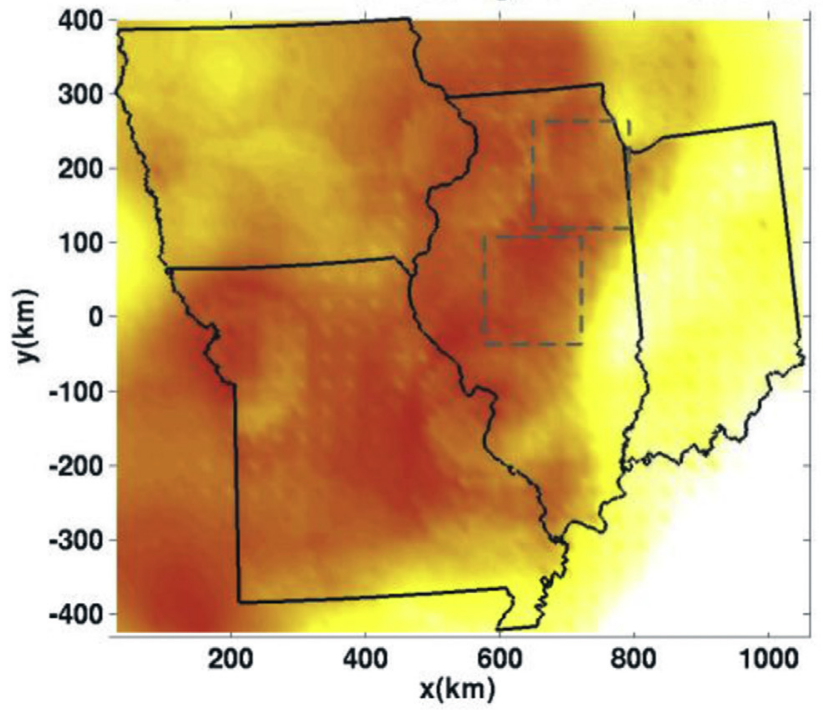

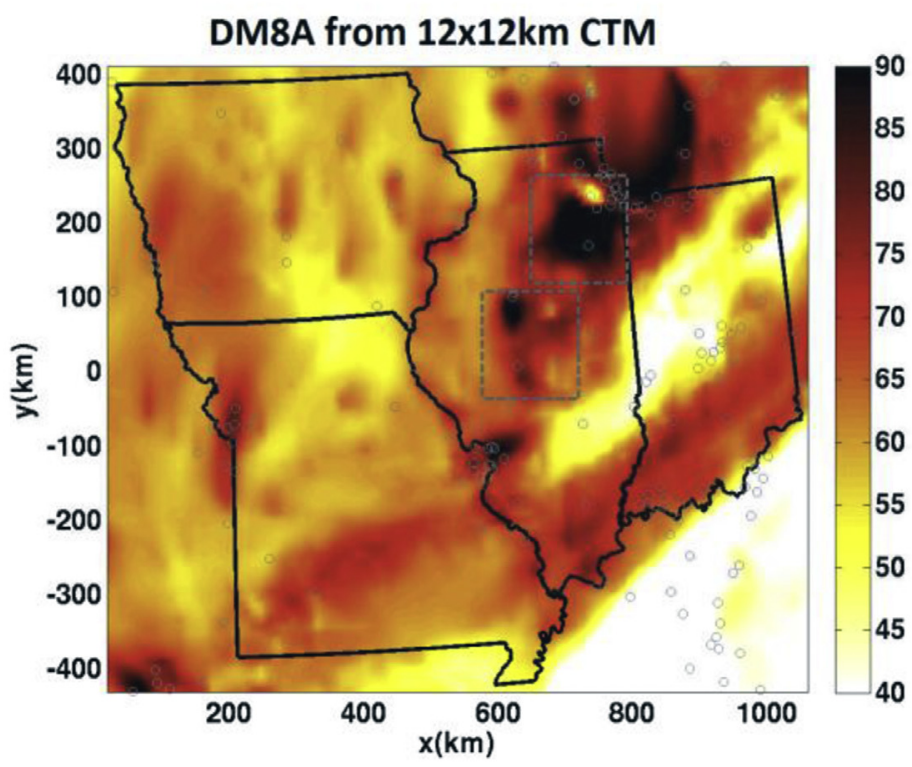

DM8A BME mean using 12x12km soft data

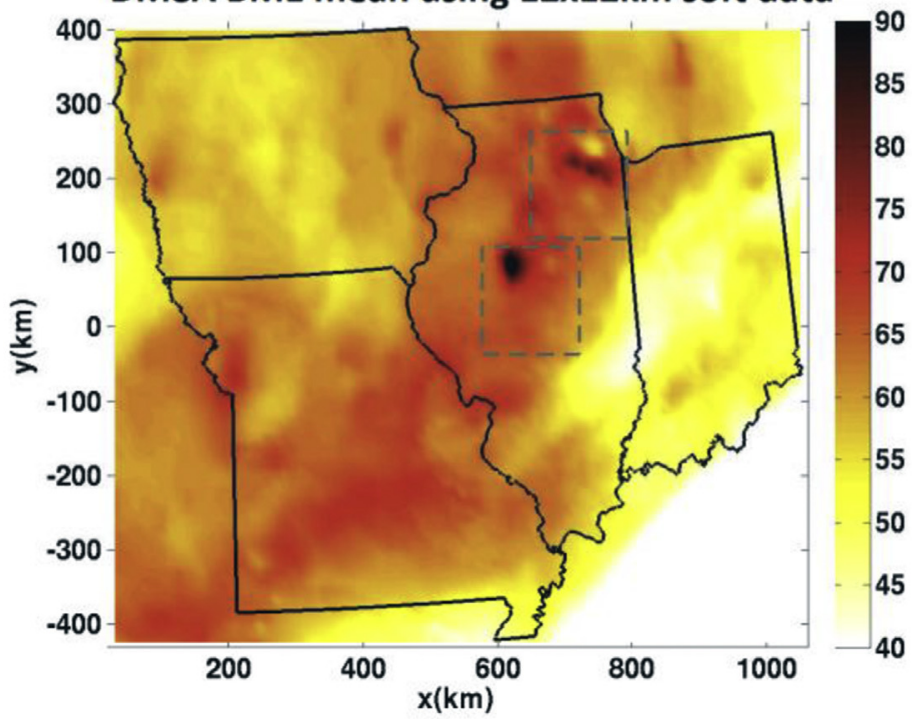

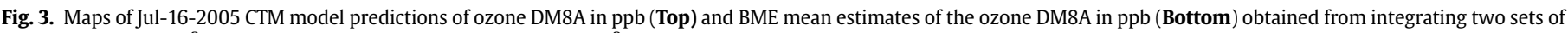

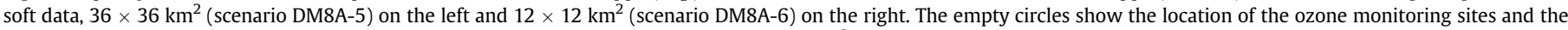
rectangle boxes marked the area of Box A and Box B (each box with the size of $144 \times 144 \mathrm{~km}^{2}$ ).

monitoring sites are not available.

\subsubsection{Validation results for daily ozone estimates}

A validation analysis on all 888 monitoring sites located within both of the CTM modeling domains was conducted to assess the influence of the CTM grid cell resolution on the accuracy of the BME estimates. Based on the cross-validation results summarized in Table 5, we see that the BME interpolation with finer grid cell resolution $\left(12 \times 12 \mathrm{~km}^{2}\right)$ resulted in an overall lower estimation error and a higher $\mathrm{R}^{2}$, when comparing scenario DM8A-6 with DM8A-5 or scenario D24A-6 with D24A-5. We also calculated the $\mathrm{R}^{2}$ and RMSE on each monitoring site to quantify the improvements contributed by using the finer grid cell resolution soft data (See maps in SI Fig. 25s and 26s). We found that, for the daily metric $\mathrm{D} 24 \mathrm{~A}$, the majority of the sites (539 out of 888 sites) have a $\mathrm{R}^{2}$ increase when using finer grid cell resolution soft data, while 676 out 
Table 4

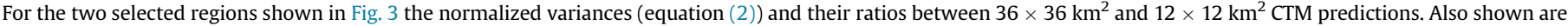
the normalized variances and their ratios for the BME mean estimates between scenarios DM8A-5 and DM8A-6.

\begin{tabular}{|c|c|c|c|c|c|c|c|}
\hline \multirow[t]{2}{*}{ Location } & \multirow[t]{2}{*}{ CTM Predictions } & & \multicolumn{5}{|c|}{ BME Mean Estimates } \\
\hline & & & $\boldsymbol{r}_{\boldsymbol{h}}(\mathrm{km})^{\mathrm{a}}$ & 0 & 36 & 72 & 108 \\
\hline \multirow[t]{3}{*}{ Box A } & NVar $_{\text {СTM_12km }}$ & 0.0107 & NVar ${ }_{\text {DM8A-6 }}$ & 0.0068 & 0.0072 & 0.0074 & 0.0075 \\
\hline & NVar $_{\text {CTM_36 }} 36 \mathrm{~km}$ & 0.0019 & NVar $_{\text {DM8A-5 }}$ & 0.0015 & 0.0014 & 0.0014 & 0.0014 \\
\hline & Ratio & 5.63 & Ratio & 4.48 & 5.04 & 5.17 & 5.36 \\
\hline \multirow[t]{3}{*}{ Box B } & NVar $_{\text {CTM_12 }}$ km & 0.0253 & NVar ${ }_{\text {DM8A-6 }}$ & 0.0051 & 0.0043 & 0.0038 & 0.0051 \\
\hline & NVar $_{\text {CTM_36 }}$ & 0.0051 & NVar & 0.0033 & 0.0019 & 0.0015 & 0.0013 \\
\hline & Ratio & 4.96 & Ratio & 1.55 & 2.23 & 2.53 & 3.92 \\
\hline
\end{tabular}

${ }^{\text {a }} \boldsymbol{r}_{\boldsymbol{h}}$ is the estimation radius around each estimation point within which all observation data are excluded in the BME integration.

Table 5

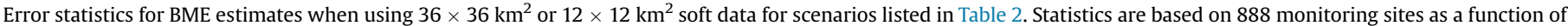
validation radius.

\begin{tabular}{|c|c|c|c|c|c|c|}
\hline \multirow[t]{2}{*}{ Output Time Average } & \multirow[t]{2}{*}{ Soft data } & \multirow[t]{2}{*}{ Error statistics } & \multicolumn{4}{|c|}{$\boldsymbol{r}_{\boldsymbol{v}}(\mathbf{k m})^{\mathrm{a}}$} \\
\hline & & & 0 & 36 & 72 & 108 \\
\hline \multirow[t]{5}{*}{ DM8A } & CTM_36k & $R M S E_{D M 8 A-5}(p p b)^{\mathrm{b}}$ & 5.202 & 5.820 & 6.238 & 6.499 \\
\hline & CTM_12k & $R M S E_{D M 8 A-6}(p p b)^{\mathrm{b}}$ & 5.113 & 5.683 & 6.088 & 6.340 \\
\hline & CTM_36k & $R_{D M 8 A-5}^{2}\left(\right.$ unitless) ${ }^{\mathrm{C}}$ & 0.901 & 0.876 & 0.860 & 0.849 \\
\hline & CTM_12k & $R_{D M 8 A-6}^{2}\left(\right.$ unitless) ${ }^{\mathrm{C}}$ & 0.905 & 0.884 & 0.868 & 0.858 \\
\hline & NA & PCR $^{2}$ DM8A-5 $\rightarrow$ DM8A-6 $(\%){ }^{d}$ & 0.44 & 0.91 & 0.93 & 1.06 \\
\hline \multirow[t]{5}{*}{ D24A } & CTM_36k & $\operatorname{RMSE}_{D 24 A-5}(p p b)^{\mathrm{b}}$ & 5.261 & 5.485 & 5.590 & 5.744 \\
\hline & CTM_12k & $R M S E_{D 24 A-6}(p p b)^{\mathrm{b}}$ & 5.037 & 5.279 & 5.373 & 5.512 \\
\hline & CTM_36k & $R_{D 24 A-5}^{2}$ (unitless) ${ }^{\mathrm{C}}$ & 0.815 & 0.798 & 0.793 & 0.782 \\
\hline & CTM_12k & $R_{D 24 A-6}^{2}\left(\right.$ unitless) ${ }^{\mathrm{C}}$ & 0.830 & 0.816 & 0.811 & 0.802 \\
\hline & $\mathrm{NA}$ & $\mathrm{PCR}^{2}$ D24A-5 $\rightarrow$ D24A-6 $(\%)^{\mathrm{d}}$ & 1.84 & 2.26 & 2.27 & 2.56 \\
\hline
\end{tabular}

${ }^{a}{ }_{v}$ is the validation radius around monitoring stations within which all observation points are excluded in the validation estimations.

b Root mean square errors.

c Squared spearman's correlation coefficients between the ozone observations and the BME estimates.

d Percent change in squared spearman's correlation coefficients from one scenario to the other.

of 888 sites have a RMSE decrease when using soft data with $12 \times 12 \mathrm{~km}^{2}$ grid cell resolution compared with the one with $36 \times 36 \mathrm{~km}^{2}$ grid cell resolution. For the daily metric DM8A, we also see a significant improvement in terms of RMSE and $\mathrm{R}^{2}$ at a majority of the sites when using soft data with finer grid cell resolution The better ozone CTM model performance in the simulation with finer grid cell resolution can explain this improvement. As we mentioned in the previous section, the CTM model predictions with a $12 \times 12 \mathrm{~km}^{2}$ grid cell resolution had a smaller prediction error and less uncertainty, which lead to a set of soft data with more informative secondary information.

\section{Discussion}

We have developed a BME framework that integrates observations from a surface monitoring network and predictions from a CTM model to create improved exposure estimates that can be resolved into any spatial and temporal resolution. The flexibility of the framework allows for input of data in any choice of time averages and CTM predictions of any spatial resolution with varying associated degrees of estimation error and cost in terms of implementation and computation. This study quantifies the impact on exposure estimation error due to these choices by first comparing estimation errors when BME relied on ozone concentration data either as an hourly average, the daily maximum 8-h average (DM8A) that is commonly used in regulatory application (Fuentes and Raftery, 2005; Nazelle et al., 2010), or the daily 24-h average (D24A) that is often used in epidemiological studies (Franklin and Schwartz, 2008; Breton et al., 2012; Bell et al., 2006).

Our analysis found that the use of DM8A and D24A data, although 200 fold less computationally intensive (Table 6s), reduced estimation error in comparison to the use of hourly observational data as BME input. The reason for this lies in the poorer model performance of the hourly CTM data when compared to DM8A or D24A averaged predictions. The BME framework was able to accurately capture this variability in CTM model performance and successfully discounted its weight in the estimation. As errors were reduced in the time weighted averaged the BME was able to rely on this data to improve estimations. The findings suggest the importance of model performance in choosing the time weighted averages used in the BME estimation. This is especially true for CTM episodes that were designed to address regulatory issues. In these situations, the focus is placed on improving the model's ability to capture peak ozone sometimes at the detriment in performance on the low ozone days, as shown in Fig. 10s and 11s the summer and winter differences in CTM model performance. In an attainment application only the predictions from the highest ozone days are used to justify regulation. As we push these CTMs applications toward exposure assessment then both high and low ozone days are used with varying degrees of performance. The BME framework is able to quantify and properly weigh the variability in this model performance. Although an increase in temporal resolution, such as hourly, is desirable, it is only useful when it is needed for relevant exposure models and if it also coincides with an improvement in model performance.

A similar finding was discovered when comparing the use of soft data processed from CTM model predictions with different grid cell resolutions. The ozone CTM model performance with a finer grid cell resolution $\left(12 \times 12 \mathrm{~km}^{2}\right)$ is better than the one with the coarser grid cell resolution $\left(36 \times 36 \mathrm{~km}^{2}\right)$. The BME framework was able to capture more spatial variability in daily ozone with the finer grid cell resolution resulting in up to a 5 times increase in normalized variances when compared with using predictions from the coarser grid cell resolution. 
Reducing the estimation error and also improving the capacity of capturing the spatial variation in ozone concentrations within a study area are two important components to reduce the exposure misclassification in epidemiologic studies. The use of observational and model predictions in a BME framework continues to be a powerful approach for improving our exposure estimates. The results of this analysis demonstrate the importance of also understanding model performance variability and its implications on exposure error. Importantly, the BME method described here has the ability to appropriately quantify the uncertainties from CTM predictions in generating soft data and apply it successfully to ozone estimations.

\section{Acknowledgments}

This work was supported by National Institute on Aging (NIA) of the National Institutes of Health (NIH) under award number R01AG033078. It was also supported by a grant (T32ES007018) from the National Institute of Environmental Health Sciences training program of NIH. The content is solely the responsibility of the authors and does not necessarily represent the official views of the NIH. The CAMx CTM modeling was performed by the U.S. Environmental Protection Agency (EPA), but this work has not been subjected to any EPA review and therefore does not necessarily reflect the views of the Agency.

\section{Appendix A. Supplementary data}

Supplementary data related to this article can be found at http:// dx.doi.org/10.1016/j.atmosenv.2017.07.033.

\section{References}

Appel, K.W., Gilliland, A.B., Sarwar, G., Gilliam, R.C., 2007. Evaluation of the community multiscale air quality (CMAQ) model version 4.5: sensitivities impacting model performance: Part I,Ozone. Atmos. Environ. 41 (40), 9603-9615.

Appel, K.W., Roselle, S.J., Gilliam, R.C., Pleim, J.E., 2009. Sensitivity of the community multiscale air quality (CMAQ) model v4.7 results for the eastern United States to MM5 and WRF meteorological drivers. Geosci. Model Dev. 2 (2), 1081-1114.

Appel, K.W., Chemel, C., Roselle, S.J., Francis, X.V., Hu, R.-M., Sokhi, R.S., Rao, S.T., Galmarini, S., 2012. Examination of the community multiscale air quality (CMAQ) model performance over the North American and European domains. Atmos. Environ. 53, 142-155.
Appel, K.W., Roselle, S., Pouliot, G., Eder, B., 2012. Performance summary of the 2006 community multiscale air quality (CMAQ) simulation for the AQMEII project: North American application. Air Pollut. Model. Appl. XXI 4, 505-511.

Bell, M.L., Peng, R.D., Dominici, F., 2006. The exposure-response curve for ozone and risk of mortality and the adequacy of current ozone regulations. Environ. Health Perspect. 114 (4), 532-536.

Breton, C.V., Wang, X., Mack, W.J., Berhane, K., Lopez, M., Islam, T.S., Feng, M., Lurmann, F., McConnell, R., Hodis, H.N., Kunzli, N., Avol, E., 2012. Childhood air pollutant exposure and carotid artery intima-media thickness in young Adults. Circulation 126 (13), 1614-1620.

EPA US. Total Risk Integrated Methodology (TRIM) - Air Pollutants Exposure Mode Documentation (TRIM.Expo/APEX, Version 4.4) Volume II: Technical Support. 2013;EPA document number EPA-452/B-12-001b..

EPA, U.S., 2014. Health risk and exposure assessment for ozone final report. https:// www3.epa.gov/ttn/naaqs/standards/ozone/data/.

Foley, K.M., Roselle, S.J., Appel, K.W., Bhave, P.V., 2009. Incremental testing of the community multiscale air quality (CMAQ) modeling system version 4.7. Geosci. Model Dev. 2 (2), 1245-1297.

Franklin, M., Schwartz, J., 2008. The impact of secondary particles on the association between ambient ozone and mortality. Environ. health Perspect. 116 (4), 453.

Fuentes, M., Raftery, A.E., 2005. Model Evaluation and spatial interpolation by bayesian combination of observations with outputs from numerical models. Biometrics 61 (1), 36-45.

Jerrett, M.P., Burnett, R.T.P., Pope, C.A.I.I.I.P., Ito, K.P., Thurston, G.S., Krewski, D.P. Shi, Y.M.D., Calle, E.P., Thun, M.M.D., 2009. Long-term ozone exposure and mortality. N. Engl. J. Med. 360 (11), 1085-1095.

Lee, S.-J., Serre, M.L., Aaron van, D., Martin, R.V., Burnett, R.T., Jerrett, M., 2012. Comparison of geostatistical interpolation and remote sensing techniques for estimating long-term exposure to ambient PM 2.5 concentrations across the continental United States. Environ. Health Perspect. 120 (12), 1727-1732.

Nazelle, Ad, Arunachalam, S., Serre, M.L., 2010. Bayesian maximum Entropy integration of ozone observations and model predictions: an application for attainment demonstration in North Carolina. Environ. Sci. Technol. 44 (15), 5707-5713.

Neidell, M., Kinney, P.L., 2010. Estimates of the association between ozone and asthma hospitalizations that account for behavioral responses to air quality information. Environ. Sci. Policy 13 (2), 97-103.

Pride, K.R., Peel, J.L., Robinson, B.F., Busacker, A., Grandpre, J., Bisgard, K.M., Yip, F.Y., Murphy, T.D., 2015. Association of short-term exposure to ground-level ozone and respiratory outpatient clinic visits in a rural location - sublette County, Wyoming, 2008-2011. Environ. Res. 137, 1-7.

Tesche, T.W., Morris, R., Tonnesen, G., McNally, D., Boylan, J., Brewer, P., 2006 CMAQ/CAMx annual 2002 performance evaluation over the eastern US. Atmos. Environ. 40 (26), 4906-4919.

Tessum, C.W., Hill, J.D., Marshall, J.D., 2015. Twelve-month, 12 km resolution North American WRF-Chem v3.4 air quality simulation: performance evaluation. Geosci. Model Dev. 8 (4), 957-973.

Xu, Y. Serre, M.L., Reyes, J. Vizuete, W., 2016. Bayesian maximum Entropy integration of ozone observations and model predictions: a national application. Environ. Sci. Technol. 50 (8), 4393-4400.

Yu S, Yu SC, Eder B, Dennis R, Chu SH. New unbiased symmetric metrics for evaluation of air quality models. Atmos. Sci. Lett.;7(1):26-34.. 